\title{
A Note on Bayesian Inference After Multiple Imputation
}

\author{
Xiang ZHOU and Jerome P. REITER
}

This article is aimed at practitioners who plan to use Bayesian inference on multiply-imputed datasets in settings where posterior distributions of the parameters of interest are not approximately Gaussian. We seek to steer practitioners away from a naive approach to Bayesian inference, namely estimating the posterior distribution in each completed dataset and averaging functionals of these distributions. We demonstrate that this approach results in unreliable inferences. A better approach is to mix draws from the posterior distributions from each completed dataset, and use the mixed draws to summarize the posterior distribution. Using simulations, we show that for this second approach to work well, the number of imputed datasets should be large. In particular, five to ten imputed datasets-which is the standard recommendation for multiple imputation-is generally not enough to result in reliable Bayesian inferences.

KEY WORDS: Missing; Nonresponse; Sample.

\section{INTRODUCTION}

When some data values are missing, one approach to statistical inference is multiple imputation (Rubin 1987; Reiter and Raghunathan 2007). The basic idea is for the analyst to fill in any missing values by repeatedly sampling from the predictive distributions of the missing values. When the posterior distribution of the parameter of interest, or, for likelihood-oriented statisticians, the sampling distribution of the complete-data estimator, is approximately Gaussian, the analyst can obtain inferences by computing point and variance estimates of interest with each dataset and combining these estimates using simple formulas. These formulas serve to propagate the uncertainty introduced by imputation through the analyst's inferences.

When presuming normality of the posterior/sampling distribution is not justifiable, the distribution is not adequately summarized by the mean and variance, so that Rubin's (1987) rules

Xiang Zhou is Ph.D. Candidate, Department of Neurobiology, and M.A., Department of Statistical Science, Duke University, Durham, NC 27708 (E-mail:x.zhou@duke.edu). Jerome P. Reiter is Associate Professor, Department of Statistical Science, Duke University, Durham, NC 27708 (E-mail: jerry@stat.duke.edu). This work was supported by the Environmental Protection Agency grant R833293. The authors thank the associate editor and a referee for suggestions on how to explain the bias in the naive approach. are not appropriate for inference. Nonetheless, some practitioners continue to use Rubin's (1987) rules even when they are theoretically invalid. For example, in a literature review of applications of multiple imputation involving parameters not adequately modeled with normal distributions, Marshall et al. (2009) found that, "Rubin's rules without applying any transformations were the standard approach used, when any method was stated." They went on to cite several examples where Rubin's (1987) rules are used to estimate functionals of distributions, such as percentiles of survival distributions.

When normality is not justifiable, Bayesian approaches are viable options for inference. In multiple imputation contexts, the analyst must appropriately utilize the information from the multiple datasets in the inferences; again, simply applying $\mathrm{Ru}$ bin's (1987) rules to posterior means and variances is generally not correct. An approach suggested by Gelman et al. (2004, p. 520) is (i) simulate many draws from the posterior distribution in each imputed dataset, and (ii) mix all the draws. The mixed draws approximate the posterior distribution. Gelman et al. (2004) did not evaluate the properties of this approximation, nor do the prominent texts on multiple imputation of Schafer (1997) and Little and Rubin (2002).

In this article, we examine the approximation of Gelman et al. (2004, p. 520) using simulation studies. We find that the approach works well with large numbers of multiply-imputed datasets. However, the usual advice for multiple imputation for modest fractions of missing data-that five or ten completed datasets are adequate for inferences - can result in unreliable estimates of posterior distributions. We also point out the pitfalls of incorrectly using Rubin's (1987) rules on functionals of posterior distributions. Specifically, we examine an approach akin to some of those observed by Marshall et al. (2009): (i) estimate posterior quantiles in each completed dataset, and (ii) average them across the datasets. We argue and demonstrate that this approach produces unreliable estimates of posterior distributions.

\section{DESCRIPTION OF THE APPROACHES}

In this section, we motivate the approach of Gelman et al. (2004, p. 520). We begin with brief reviews of Bayesian inference with incomplete data and of multiple imputation. Let $Y_{\text {inc }}=\left(Y_{o b s}, Y_{\text {mis }}\right)$ be the $n \times p$ matrix of data values for $n$ units, where $Y_{o b s}$ is the portion of $Y_{\text {inc }}$ that is observed, and $Y_{m i s}$ is the portion of $Y_{\text {inc }}$ that is missing. Let $Q$ be a parameter of interest, for example a regression coefficient. Let $Q_{\alpha}$ be the value of $Q$ 
such that

$$
\begin{aligned}
& \int_{-\infty}^{Q_{\alpha}} f\left(Q \mid Y_{o b s}\right) d Q \\
& \quad=\int_{-\infty}^{Q_{\alpha}} \int_{Y_{m i s}} f\left(Q \mid Y_{o b s}, Y_{m i s}\right) f\left(Y_{m i s} \mid Y_{o b s}\right) d Y_{m i s} d Q \\
& \quad=\alpha,
\end{aligned}
$$

where $\alpha$ is a desired quantile of the posterior distribution of $Q$. Analysts can approximate this integral with Monte Carlo methods. First, draw $Y_{m i s}$ from its posterior predictive distribution. Second, draw a value of $Q$ from its posterior distribution, given the drawn $Y_{m i s}$. Third, repeat these two steps $K$ times, where $K$ is very large. Fourth, sort the $K$ simulated values of $Q$, and select the $(\alpha K)$ th element of the sorted list. The result is an estimate for $Q_{\alpha}$.

In multiple imputation, the analyst creates $m$ completed datasets, $D^{(l)}=\left(Y_{o b s}, Y_{m i s}^{(l)}\right)$ where $1 \leq l \leq m$, which are used for analysis. Here, $Y_{m i s}^{(l)}$ is a draw from the posterior predictive distribution of $\left(Y_{m i s} \mid Y_{o b s}\right)$, or from an approximation of that distribution such as the approach of Raghunathan et al. (2001).

Typically, $m$ is much smaller than $K$ would be for Bayesian inference for non-Gaussian distributions. Thus, with small $m$, drawing one value of $Q$ for each $D^{(l)}$ results in too few draws of $Q$ to get reasonable estimates of $Q_{\alpha}$. Instead, we can utilize each completed dataset for more than just one draw of $Q$. To motivate this, we re-express the integral in (1) as

$$
\alpha=\lim _{m \rightarrow \infty} \frac{1}{m} \sum_{l=1}^{m} \int_{-\infty}^{Q_{\alpha}} f\left(Q \mid Y_{o b s}, Y_{m i s}^{(l)}\right) d Q .
$$

This suggests that, for any value of $Q$ in the upper limit of the integral, we can find the associated cumulative probability by (i) sampling $J$ values of $Q$ in each $D^{(l)}$, where $J$ is large, (ii) finding the percentage of the $J$ draws in each $D^{(l)}$ less than the upper limit value, and (iii) averaging those percentages across all $m$ datasets, where $m \rightarrow \infty$. Equivalently, the analyst could mix all of the sampled draws from each dataset, and find the percentage of elements less than the upper limit in the combined draws. This process can be easily adapted to find $Q_{\alpha}$ : try different upper limits until one reaches the desired $\alpha$ probability.

The approximation of Gelman et al. (2004, p. 520), which we denote as $\tilde{Q}_{\alpha}$, is essentially an approximation of (2) for finite $m$. Specifically, for each $D^{(l)}$ where $l=1, \ldots, m$, the analyst simulates $J$ values of $Q$ from $f\left(Q \mid D^{(l)}\right)$, where $J$ is large. Let $\hat{f}\left(Q^{(l)}\right)$ represent the $J$ draws of $Q$ obtained with $D^{(l)}$. The analyst mixes all $\hat{f}\left(Q^{(l)}\right)$ together to create $\hat{f}\left(Q^{\text {all }}\right)$. The analyst sorts the $m J$ draws in $\hat{f}\left(Q^{\text {all }}\right)$, and the $\alpha(m J)$ th element of the sorted list is the estimate of $Q_{\alpha}$.

We now use simulations to illustrate the properties of $\tilde{Q}_{\alpha}$. We also use the simulations to emphasize that the naive approach of averaging posterior quantiles can produce poor estimates of $Q_{\alpha}$ in comparison to $\tilde{Q}_{\alpha}$. To fix notation for the naive approach, let $Q_{\alpha}^{(l)}$ be the value of $Q$ in $D^{(l)}$ such that $\int_{-\infty}^{Q_{\alpha}^{(l)}} f\left(Q \mid Y_{o b s}, Y_{m i s}^{(l)}\right) d Q=\alpha$. Then, $\bar{Q}_{\alpha}=\sum_{l=1}^{m} Q_{\alpha}^{(l)} / m$. Clearly, $\bar{Q}_{\alpha}$ has nothing to do with (2). It is derived from convenience rather than theory.

\section{ILLUSTRATIVE SIMULATIONS}

The complete data, $Y_{i n c}$, comprise $n=50$ values generated independently from Bernoulli trials with $\pi=0.2$. We introduce missing data by randomly deleting $10 \%, 30 \%$, or $50 \%$ of the data completely at random (Rubin 1976). We use multiple imputation to generate $m=5, m=20$, or $m=100$ completed datasets. We seek $Q_{\alpha}$, where $\alpha \in\{0.025,0.25,0.75,0.975\}$, for the posterior distribution of $\pi$. We generate $Y_{i n c}$ and multiple imputations 5000 times to approximate the sampling distributions of $\tilde{Q}_{\alpha}$ and $\bar{Q}_{\alpha}$.

To create each completed dataset, we first sample a value of $\pi$ from the appropriate Beta distribution based on $Y_{o b s}$. We use a uniform prior distribution for $\pi$. We then draw $Y_{m i s}$ from a Bernoulli distribution using the sampled $\pi$. After the imputation steps, in each $D^{(l)}$ we draw $J=10,000$ values of $\pi$ from $\operatorname{Beta}\left(\sum\left(Y_{o b s}+Y_{m i s}^{(l)}\right)+1, n-\sum\left(Y_{o b s}+Y_{m i s}^{(l)}\right)+1\right)$, which is the posterior computed with $D^{(l)}$. To get $\tilde{Q}_{\alpha}$, we mix and sort the $m J$ draws of $\pi$, and select the $\alpha(m J)$ th element. To get $\bar{Q}_{\alpha}$, we compute the $\alpha$-quantile in each $D^{(l)}$ and average them across the $m$ datasets.

Figure 1 shows the distributions of $\tilde{Q}_{\alpha}-Q_{\alpha}$ and $\bar{Q}_{\alpha}-Q_{\alpha}$ for $\alpha \in\{0.025,0.25\}$ across the 5000 replications with $m=$ 100 . Here, $Q_{\alpha}$ is computed from $f\left(\pi \mid Y_{o b s}\right)$. For each scenario, $\tilde{Q}_{\alpha}$ is nearly centered on $Q_{\alpha}$. There do not appear to be any trends with the percentage of missing data, apart from the expected increase in variability as the percentage of missing data increases. However, in additional simulations with $m=5$ and $m=20$, typically $\tilde{Q}_{\alpha}>Q_{\alpha}$ for small $\alpha$, and $\tilde{Q}_{\alpha}<Q_{\alpha}$ for large $\alpha$. This is evident in Figure 2, which shows that when $m=5$ and to a lesser extent when $m=20$, the posterior intervals based on $\tilde{Q}_{\alpha}$ tend to be tighter than warranted for modest $m$. This problem disappears when $m=100$.

The inaccuracy when $m=5$ merits closer inspection, because often practitioners only create five multiple imputations for analysis. Across all missing-data scenarios, the median lengths of the $50 \%$ and $95 \%$ posterior intervals are smaller when $m=5$ than when $m=100$. Put another way, analysts appear to obtain sharper inferences by using five imputations than using one hundred imputations. This does not imply that analysts should use small $m$ for Bayesian inference after multiple imputation; on the contrary, it implies that approximations $\tilde{Q}_{\alpha}$ based on small $m$ are not reliable. Hence, analysts planning on Bayesian inference after multiple imputation should generate a large number of completed datasets.

What about $\bar{Q}_{\alpha}$ ? As evident in Figure $1, \bar{Q}_{\alpha}$ can differ substantially from $Q_{\alpha}$, and its performance worsens as the percentage of missing values increases. More often than not, $\bar{Q}_{\alpha}>Q_{\alpha}$ for small $\alpha$, and $\bar{Q}_{\alpha}<Q_{\alpha}$ for large $\alpha$. Hence, as also evident in Figure 2, analysts who construct posterior intervals based on $\bar{Q}_{\alpha}$ tend to have tighter ranges than warranted.

What is wrong with the naive approach of averaging posterior quantiles? Each $Q_{\alpha}^{(l)}$ is a summary of the posterior distribution of $Q$ estimated as if $D^{(l)}$ was in fact genuine data with $n$ records. However, the observed data comprise fewer than $n$ records, so that the actual posterior distribution of $Q$ is more dispersed than the complete-data posterior distribution. Thus, each $Q_{\alpha}^{(l)}$ is biased toward the median, and so is their average. 

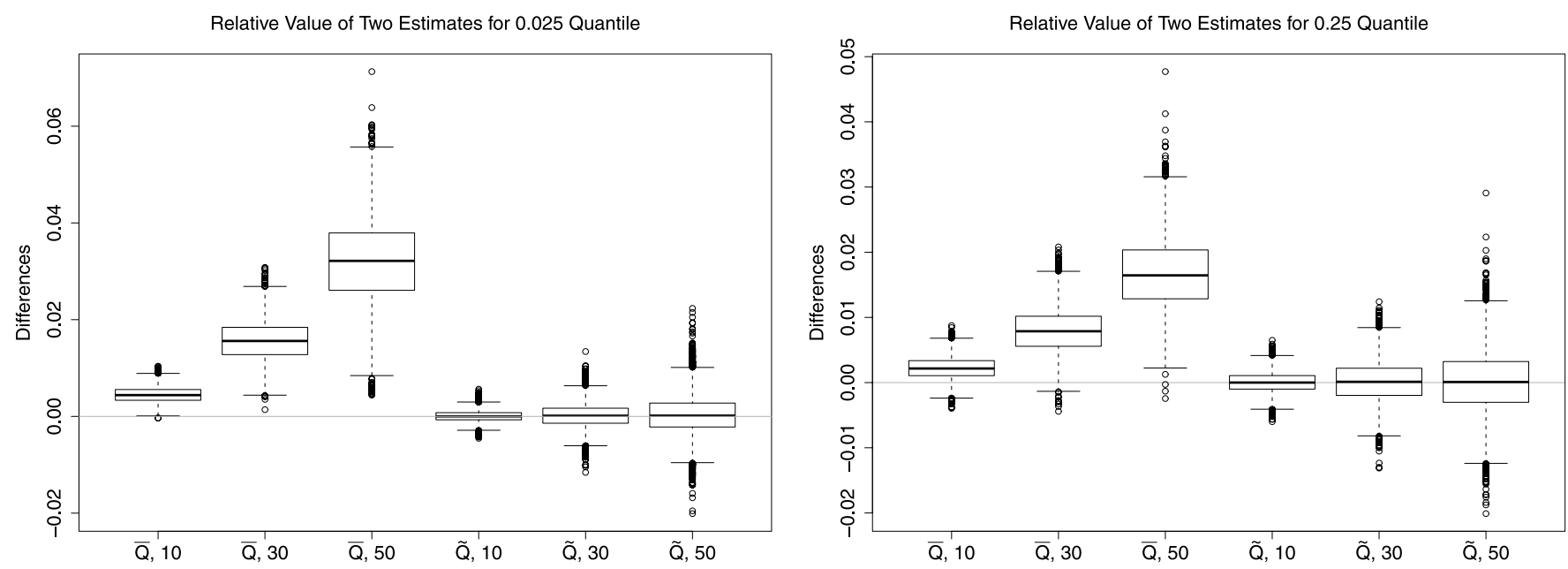

Figure 1. Boxplots of $\bar{Q}_{\alpha}-Q_{\alpha}$ and $\tilde{Q}_{\alpha}-Q_{\alpha}$ in different settings with $n=50, m=100$, and $10 \%$, 30\%, or 50\% missing data. The first three plots in each panel are for $\bar{Q}_{\alpha}-Q_{\alpha}$, and the second three plots in each panel are for $\tilde{Q}_{\alpha}-Q_{\alpha}$. The labels on the horizontal axis show the percentage of missing data. Generally, $\bar{Q}_{\alpha}$ is substantially different than $Q_{\alpha}$, whereas $\tilde{Q}_{\alpha}$ estimates $Q_{\alpha}$ reasonably well.
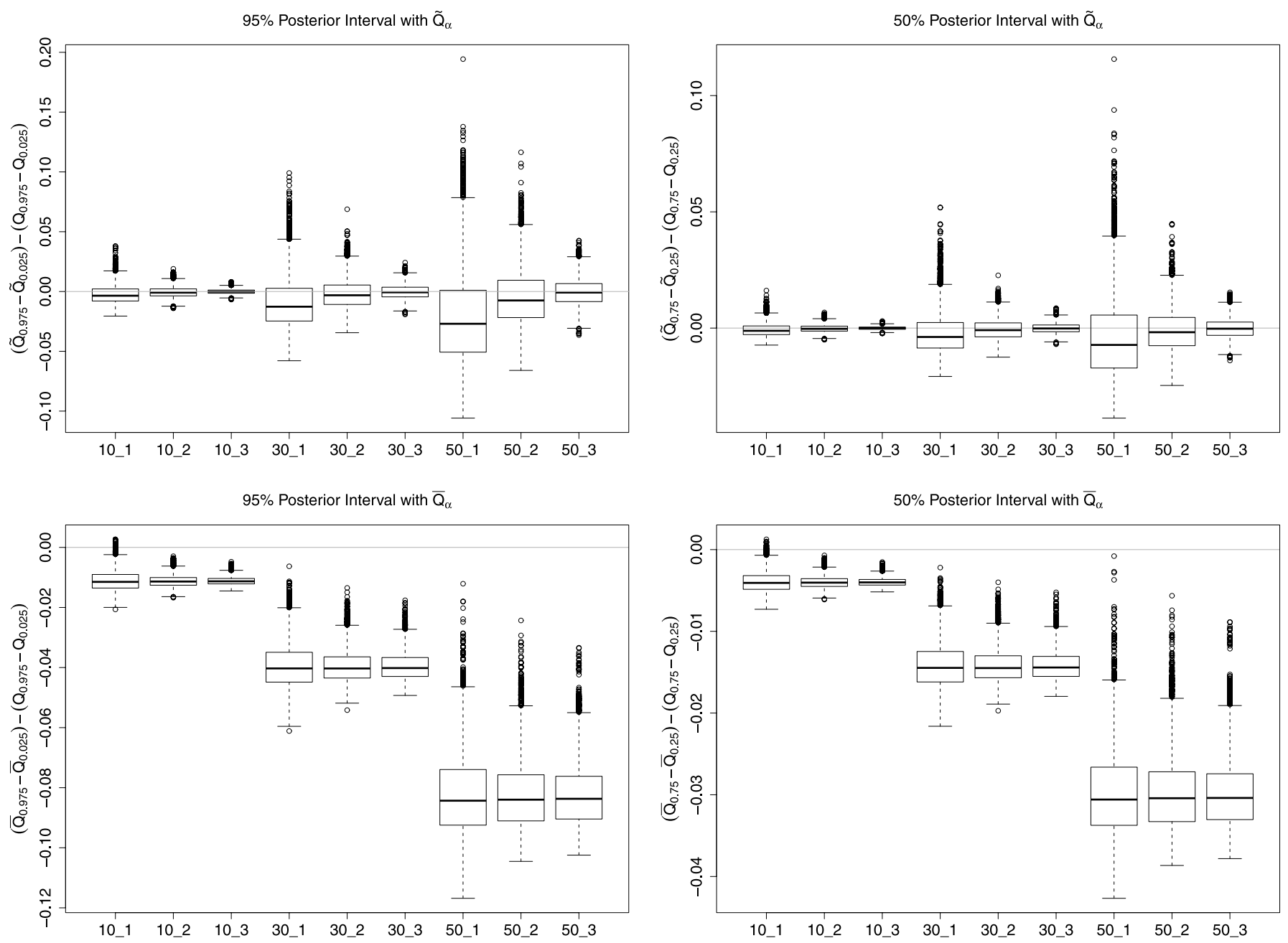

Figure 2. Boxplots of differences in lengths of the approximate and true $50 \%$ and $95 \%$ posterior intervals with $n=50$. The labels on the horizontal axis show the percentage of missing data followed by the value of $m$, where 1 represents $m=5,2$ represents $m=20$, and 3 represents $m=100$. The intervals based on $\tilde{Q}_{\alpha}$ (top panel) are relatively poor approximations for modest $m$ but good for large $m$. The intervals based on $\bar{Q}_{\alpha}$ (bottom panel) are always unreliable. 


\section{APPLICATION TO BIOASSAY DATA}

To illustrate Bayesian inference after multiple imputation on genuine data, we modify data from a bioassay experiment that appears in the book by Gelman et al. (2004, pp. 88-93), who took them from Racine et al. (1986). The data comprise two measurements on $n=20$ animals. Let $x_{i}$ be the natural logarithm of the dose of a chemical compound administered to animal $i$, where $x_{i} \in\{-0.86,-0.30,-0.05,0.73\}$. There were five animals at each dose level. Let $y_{i}=1$ if animal $i$ dies shortly after receiving the dose, and let $y_{i}=0$ otherwise. There are no missing data in the study. Therefore, we deleted a randomly selected $20 \%$ of the $y_{i}$ values.

The goal of the analysis is to learn about the toxicity of the compound, which we do with a logistic regression of $Y$ on $X$. Because of the small sample size, it is doubtful that the sampling distributions of the estimated regression coefficients are well-approximated by normal distributions. Following Gelman et al. (2004), we therefore use a Bayesian logistic regression model to learn about the toxicity of the compound, so that $y_{i} \mid \pi_{i} \sim \operatorname{Bernoulli}\left(\pi_{i}\right)$ where $\operatorname{logit}\left(\pi_{i}\right)=\beta_{0}+\beta_{1} x_{i}$. We use the noninformative prior distribution $f\left(\beta_{0}, \beta_{1}\right) \propto 1$. The primary targets of scientific interest are the posterior distributions of $\beta_{0}$ and $\beta_{1}$.

To multiply-impute the four missing values, we first draw a value of $\left(\beta_{0}, \beta_{1}\right)$ from its approximate posterior distribution using grid sampling. We substitute the drawn values into the equation for $\pi_{i}$ for each of the four animals with missing data. We then draw values of $Y_{m i s}$ from each animal's Bernoulli distribution to create the completed dataset, $D^{(l)}$, where $l=1, \ldots, m$. We examine three scenarios: $m=5, m=20$, and $m=100$.

For each $D^{(l)}$, we determine quantiles of $f\left(\beta_{0}, \beta_{1} \mid D^{(l)}\right)$ by using grid sampling again. We sample $J=10,000$ values from the joint distribution for each completed dataset. By mixing the $m J$ draws of $\left(\beta_{0}, \beta_{1}\right)$, we can compute values of $\tilde{Q}_{\alpha}$.

Table 1 displays several quantiles for $\beta_{0}$ and $\beta_{1}$. When $m=$ 100 , the values of $\tilde{Q}_{\alpha}$ are close to the corresponding values of $Q_{\alpha}$. As expected, the differences between $\tilde{Q}_{\alpha}$ and $Q_{\alpha}$ are largest when $m=5$. For both $\beta_{0}$ and $\beta_{1}$, the posterior intervals are too narrow when $m=5$. The table also displays estimates based on $\bar{Q}_{\alpha}$. Once again, they are less reliable than those based on $\tilde{Q}_{\alpha}$.

To see if the results in Table 1 for $m=5$ are unusual, we repeated the posterior simulation 100 times. In $57 \%$ of these replications, $\tilde{Q}_{0.975}-\tilde{Q}_{0.025}$ for $\beta_{1}$ with $m=5$ was shorter than $Q_{0.975}-Q_{0.025}$ for $\beta_{1}$ from the observed data; roughly the same trend held for the interquartile range for $\beta_{1}$ and for the intervals involving $\beta_{0}$. The lengths of the one hundred $\tilde{Q}_{0.975}-\tilde{Q}_{0.025}$ for $\beta_{1}$ with $m=5$ ranged from 15.8 (1.5 to 17.3) to 21.4 (3.0 to 24.4), as compared to a length of 19.5 for $Q_{0.975}-Q_{0.025}$. Thus, there are substantial chances of estimating inappropriately short posterior intervals with $\tilde{Q}_{0.975}-\tilde{Q}_{0.025}$, although the risks appear to be random rather than systematic. Given the potential for overstating accuracy, we would be reluctant to recommend or use $m=5$ for this analysis.

We also repeated the analysis using an imputation model that differs from the analysis model. Specifically, for imputations we assume that $f\left(y_{i j}\right) \sim \operatorname{Bernoulli}\left(\pi_{j}\right)$ in each of the $j=1, \ldots, 4$ dosage strata. The results show the themes seen in Table 1.

\section{CLOSING REMARKS}

As both multiple imputation and Bayesian inference grow in popularity, we anticipate that practitioners will commonly use Bayesian inference after multiple imputation. We hope that this article reduces the number of practitioners who naively and incorrectly average posterior quantiles and other functionals, and encourages practitioners to use the approach of Gelman et al. (2004, p. 520) with large $m$.

\section{[Received May 2009. Revised January 2010.]}

\section{REFERENCES}

Gelman, A., Carlin, J. B., Stern, H. S., and Rubin, D. B. (2004), Bayesian Data Analysis, London: Chapman \& Hall. [159,160,162]

Little, R. J. A., and Rubin, D. B. (2002), Statistical Analysis With Missing Data (2nd ed.), New York: Wiley. [159]

Table 1. Quantile estimates for the bioassay data from one set of multiple imputations using $m=5, m=20$, and $m=100$. The imputation model is the same as the analysis model. Here, $Q_{\alpha}$ is estimated with the observed data. Inferences based on $\tilde{Q}_{\alpha}$ are reliable with $m=100$ but less so with $m=5$ or $m=20$. Inferences based on $\tilde{Q}_{\alpha}$ are generally unreliable.

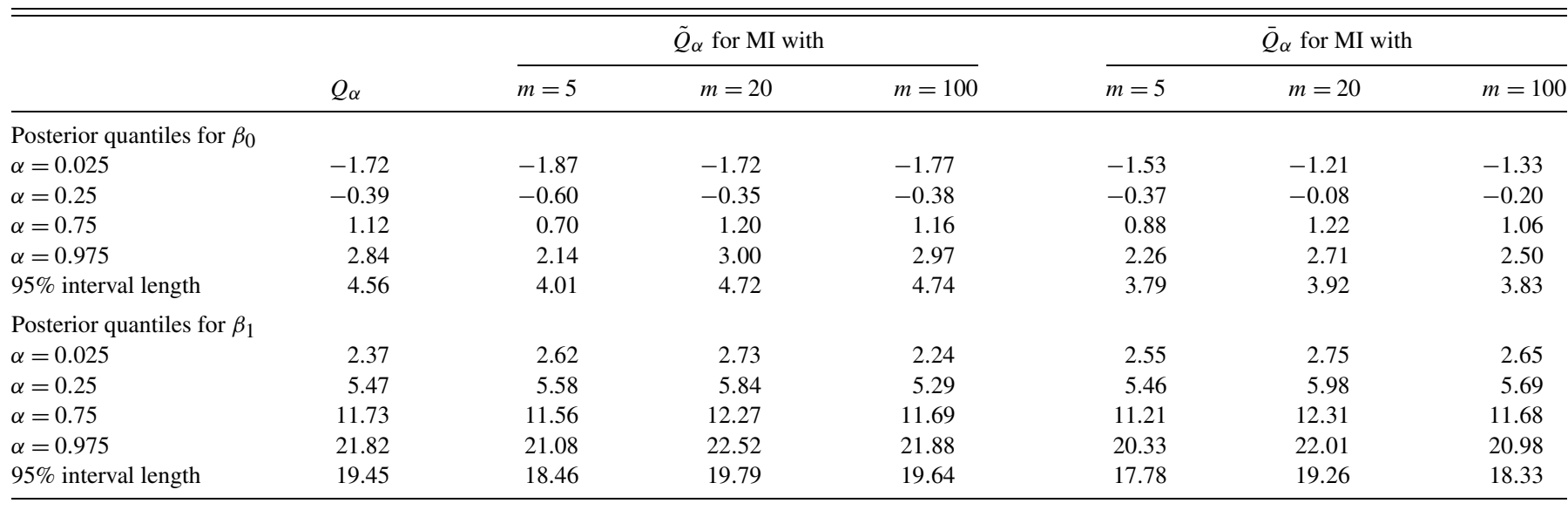


Marshall, A., Altman, D. G., Holder, R. L., and Royston, P. (2009), "Combining Estimates of Interest in Prognostic Modelling Studies After Multiple Imputation: Current Practice and Guidelines," BMC Medical Research Methodology, 9, 57. [159]

Racine, A., Grieve, A., Fluhler, H., and Smith, A. (1986), "Bayesian Methods in Practice: Experiences in the Pharmaceutical Industry" (with discussion), Applied Statistics, 35, 93-150. [162]

Raghunathan, T. E., Lepkowski, J. M., van Hoewyk, J., and Solenberger, P. (2001), "A Multivariate Technique for Multiply Imputing Missing Values Using a Series of Regression Models," Survey Methodology, 27, 85-96. [160]
Reiter, J. P., and Raghunathan, T. E. (2007), "The Multiple Adaptations of Multiple Imputation," Journal of the American Statistical Association, 102, 1462-1471. [159]

Rubin, D. B. (1976), "Inference and Missing Data" (with discussion), Biometrika, 63, 581-592. [160]

(1987), Multiple Imputation for Nonresponse in Surveys, New York: Wiley. [159]

Schafer, J. L. (1997), Analysis of Incomplete Multivariate Data, London: Chapman \& Hall. [159] 